International Scientific Organization

http://iscientific.org/

Chemistry International

www.bosaljournals.com/chemint/

\title{
An advanced trick to calculate the molecules and ions bond order with practical application
}

\author{
Mohd. Suhail
}

\begin{abstract}
Department of Chemistry, Jamia Millia Islamia (A Central University), Jamia Nagar, 110025, New Delhi, Delhi, India
*Corresponding author's E. mail: Suhailchem.786@gmail.com; mohd.suhail159068@st.jmi.ac.in
\end{abstract}

\section{A R T I C L E I N F O}

\section{Article type:}

Short communication

Article history:

Received December 2020

Accepted May 2021

July 2021 Issue

Keywords:

Bond order

Molecular orbital theory

Bonding electrons

Antibonding electrons

Advanced trick

\section{A B S T R A C T}

Every molecule has two or more atoms linked to each other through a bond. The number of bonds between two atoms is called bond order. Hence, the bond order gives information about the total number of bonds between two atoms. Besides, different methods and definitions have been given to find out an exact bond order based on various theories. The most acceptable theory to find an exact bond order is the molecular orbital theory. Using this theory, the whole electronic configuration of the molecule is written first, then total electrons present in bonding orbitals as well as antibonding orbitals are counted. After that, the bond order is calculated using an old and time-consuming formula. The presented paper describes an advanced, easy, and time-saving method, named as an advanced trick/method, with a new formula to find out an exact bond order. In this trick, only total electrons and the number of antibonding electrons is considered to calculate the bond order using developed strategy with practical examples.

(c) 2021 International Scientific Organization: All rights reserved.

Capsule Summary: The presented paper describes an easy method to find out the total number of bonds, i.e., bond order in a molecule or ion.

Cite This Article As: M. Suhail. An advanced trick to calculate the molecules and ions bond order with practical application. Chemistry International 7(3) (2021) 208-211.

https://doi.org/10.5281/zenodo.4900095

\section{INTRODUCTION}

In chemistry, there are many concepts to be made ease in calculations related to chemical functions (Ali et al, 2017a; Suhail and Ali 2020a; Suhail 2020b; 2020c). The calculation for bond order is one of them. Basically, bond order is the number of bonds present between two atoms in a molecule. In molecules having resonance or nonclassical bonding, bond numbers may not be an integer. In benzene, the delocalized molecular orbitals contain 6 pi electrons over six carbons essentially yielding half a pi bond together with the sigma bond for each pair of carbon atoms, giving a calculated bond number of 1.5. Bond order does not cause any change in the stereochemistry of carbon atom (Alajmi et al., 2016; (Ali, et al., 2016a; 2016b; 2016c; 2017b; 2017c; 2017d; 2018a; 2018b; 2018c; 2019; 2020; 2021a; 2021b).

Furthermore, bond numbers of 1.1 , for example, can arise under complex scenarios and essentially refer to bond strength relative to bonds with order 1 . Many definitions have been given to define bond order. Some scientists have given a formula-based definition, while someone described it as an index of bond strength (March, 1985). On the other hand, it has also been defined as the stability of a molecule in some textbooks. Some other old definitions about the bond order are as follows (i-iv). 


$$
\sigma 1 s<\sigma^{*} 1 s<\sigma 2 s<\sigma^{*} 2 s<\left(\pi 2 p_{x}=\pi 2 p_{y}\right)<\sigma 2 p_{z}<\left(\pi * 2 p_{x}=\pi * 2 p_{y}\right)<\sigma^{*} 2 p_{z} \text { Upto N2 }
$$

$$
\sigma 1 s<\sigma^{*} 1 s<\sigma 2 s<\sigma^{*} 2 s<\sigma 2 p_{z}<\left(\pi 2 p_{x}=\pi 2 p_{y}\right)<\left(\pi^{*} 2 p_{x}=\pi^{*} 2 p_{y}\right)<\sigma^{*} 2 p_{z} \text { Above } N_{2}
$$

(b)

Fig. 1: Orbitals filling pattern, (a) up to 14 electrons and (b) above 14 electrons

$$
\text { B.O. }=\frac{\text { number of bonding electrons }- \text { number of antibonding electrons }}{2}
$$

Fig. 2: An old formula to find out a bond order

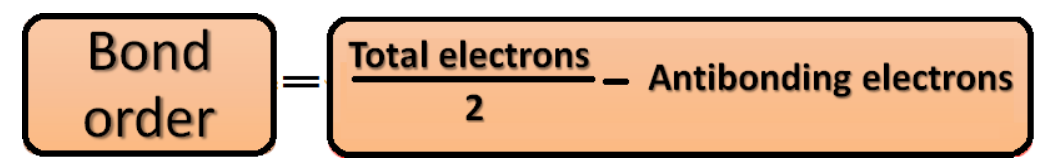

Fig. 3: Formula to find out a bond order

i. Bond order, as introduced by Linus Pauling, is defined as the difference between the number of bonds and antibonds.

ii. The bond number itself is the number of electron pairs (bonds) between a pair of atoms (IUPAC). For example, in diatomic nitrogen $\mathrm{N} \equiv \mathrm{N}$ the bond number is 3 , in acetylene $\mathrm{H}-\mathrm{C} \equiv \mathrm{C}-\mathrm{H}$ the bond number between the two carbon atoms is also 3 , and the $\mathrm{C}-\mathrm{H}$ bond order is 1 .

iii. Bond order gives an indication of the stability of a bond. Isoelectronic species have the same bond number.

iv. In molecular orbital theory, bond order is defined as half of the difference between the number of bonding electrons and the number of antibonding electrons as per the formula given in Fig. 2 (Clayden et al., 2012; Housecroft and Sharpe, 2012).

\section{An old method to find a bond order}

As per molecular orbital theory, every molecule has its own molecular orbitals and a definite electronic configuration as well. Hence, the bond order is obtained by following the steps which are as follows:

i. A correct electronic configuration is written first for a given molecule in which the bond order is to be found.

ii. The molecular energy orbitals are filled according to Afbau principle (Fig. 1a and 1b). iii. After filling electrons in the different molecular orbitals, all the electrons present in bonding and antibonding orbitals are counted.

iv. In the last step, bond order is calculated with the help of a formula given in fig. 2 .

Hence, there are four steps involved in finding of bond order.

\section{MATERIAL AND METHODS}

\section{A new method for bond order calculation}

I have given a new method with a new formula to find out an exact bond order in a molecule in a very short time. Based on formula given in (fig. 3), the bond order can be defined as the difference between half of the total electrons and antibonding electrons. For applying this method, the total number of electrons present in the molecule as well as antibonding orbitals, should be known. Antibonding electrons in a molecule are identified using Fig. $1 \mathrm{a}$ and $1 \mathrm{~b}$. Therefore, if the presented method is being followed, no need to,

i. Write the total bonding electrons.

ii. remember an especial case given in Fig. 1a and $1 \mathrm{~b}$.

iii. remember an old formula.

iv. remember an old trick.

Application of developed trick for bond order calculation 
Table 1: Calculated values of bond orders of different molecules and ions using proposed trick

\begin{tabular}{cccc}
\hline Molecules/ions & Total electrons & Antibonding electrons & Calculated bond order \\
\hline $\mathrm{H}_{2}{ }^{+}$ & 1 & 0 & 0.5 \\
$\mathrm{H}_{2}$ & 2 & 0 & 1 \\
$\mathrm{H}_{2} \mathrm{He}_{2} \mathrm{He}^{+}$ & 3 & 1 & 0.5 \\
$* \mathrm{He}_{2}$ & 4 & 2 & 0 \\
$\mathrm{Li}_{2}{ }^{+}$ & 5 & 2 & 0.5 \\
$\mathrm{Li}_{2}$ & 6 & 2 & 1 \\
$\mathrm{Li}_{2}, \mathrm{Be}_{2}{ }^{+}$ & 7 & 3 & 0.5 \\
$* \mathrm{Be}_{2}$ & 8 & 4 & 0 \\
$\mathrm{~B}_{2^{+}}$ & 9 & 4 & 0.5 \\
$\mathrm{~B}_{2}$ & 10 & 4 & 1 \\
$\mathrm{~B}_{2}$ & 11 & 4 & 1.5 \\
$\mathrm{C}_{2}$ & 12 & 4 & 2 \\
$\mathrm{~N}_{2}{ }^{+}, \mathrm{CO}^{+}$etc. & 13 & 4 & 2.5 \\
$\mathrm{~N}_{2}, \mathrm{CN}^{-}$etc. & 14 & 4 & 3 \\
$\mathrm{O}_{2}$ & 15 & 5 & 2.5 \\
$\mathrm{O}_{2}$ & 16 & 6 & 2 \\
$\mathrm{O}_{2}$ & 17 & 7 & 1.5 \\
$\mathrm{O}_{2}{ }^{2-}, \mathrm{F}_{2}$ & 18 & 8 & 1 \\
$\mathrm{~F}_{2}$ & 19 & 9 & 0.5 \\
$* \mathrm{~F}_{2}{ }^{-2}$ & 20 & 10 & 0 \\
\hline
\end{tabular}

* not exist because of zero bond order

The most important point during the calculation for bond order was the comparison of both methods, i.e., old method and new one. This comparison clearly showed that the time taken was very shorter as compared to the old method after the calculation for bond order in a molecule using the presented trick. Hence, the presented trick makes the calculation to find out an exact bond order in a molecule/ion easy. Besides, another point noted after calculation, was the similarity in the answers which makes the presented trick reliable.

\section{CONCLUSIONS}

The calculation to find out bond order has been made easy and simple in the presented method without writing the whole electronic configuration. Besides, this method saves time and also gives an exact value of bond order. The presented method of calculating bond order with a new definition, will be useful to the scientific community. The proposed method of finding bond order will be valuable in the learning any process/system, as it is a fast and easy method.

\section{ACKNOWLEDGEMENTS}

I am very thankful to my guide, Professor Imran Ali, Chemistry Department, Jamia Millia Islamia (A Central
University), New Delhi, Delhi, India for guiding and supporting morally.

\section{REFERENCES}

Alajmi, M.F., Afzal, H., Suhail, M., Sofi, D.M., Dibya, R.S., Leonid, A., Imran, A., 2016. Chiral HPLC separation and modeling of four stereomers of DL-leucine-DLtryptophan dipeptide on amylose chiral column. Chirality 28(9), 642-648.

Ali I., Suhail, M., Basheer A., 2017a. Advanced spiral periodic classification of the elements. Chemistry International 3(4), 219-223.

Ali, I, Suhail, M., Mohammad, N.L., Alothman, Z.A., Abdulrahman, A., 2016c. Chiral resolution of multichiral center racemates by different modalities of chromatography. Journal of Liquid Chromatography and Related Technologies 39(9), 435-444.

Ali, I, Suhail, M., Zeid, A.A., Ahmad, Y.B., 2018b. Stereoselective interactions of profen stereomers with human plasma proteins using nano solid phase micro membrane tip extraction and chiral liquid chromatography. Separation and Purification Technology 197, 336-344.

Ali, I., Mohammad, N.L., Suhail, M., AL-Othman, Z.A., Abdulrahman, A., 2016a. Enantiomeric resolution and simulation studies of four enantiomers of 5-bromo- 
3ethyl-3-(4-nitrophenyl)-piperidine-2,6-dione on a Chiralpak IA column. RSC Advances 6(17), 14372-14380.

Ali, I., Suhail, M., Asnin, L., Hassan, Y.A., 2020. Effect of various parameters and mechanism of reversal order of elution in chiral HPLC. Current Analytical Chemistry 16, 59-78.

Ali, I., Suhail, M., Hassan, Y.A., 2018a. Chiral Analysis of Macromolecules. Journal of Liquid Chromatography and Related Technologies 41(11), 749-760.

Ali, I., Suhail, M., Hassan, Y.A., 2019. Advances in chiral multidimensional liquid chromatography. TrAC Trends in Analytical Chemistry 120, 115634.

Ali, I., Suhail, M., Leonid, A., 2017c. Chiral separation of quinolones by liquid chromatography and capillary electrophoresis. Journal of Separation Science 40(14), 2863-2882.

Ali, I., Suhail, M., Leonid, A., 2018c. Chiral Separation and modeling of quinolones on teicoplanin macrocyclic glycopeptide antibiotics CSP. Chirality 30 (12), 13041311.

Ali, I., Suhail, M., Leonid, A., Hassan, Y.A., 2017d. Reverse elution order of $\beta$-blockers in chiral separation. Journal of Liquid Chromatography and Related Technologies 40(9), 435-441.

Ali, I., Suhail, M., Zeid A. A., Alwarthan, A., 2017b. Chiral separation and modeling of baclofen, bupropion, and etodolac profens on amylose reversed phase chiral column. Chirality 29(7), 386-397.

Ali, I., Suhail, M., Zeid, A.A., Abdulrahman, A., Hassan, Y.A., 2016b. Enantiomeric resolution of multiple chiral centres racemates by capillary electrophoresis. Biomedical Chromatography 30(5), 683-694.

Ali, I., Suhail, M., Aboul-Enein, H.Y., Kon'kova, T., 2021 a. Recent Trends in Chiral Separations by 2D-HPLC. Chromatographia 84, 535-548.

ALOthman, Z.A., Badjah, A., Alsheetan, A.Y.K.M., Suhail, M., Ali, I., 2021b. Enantiomeric resolution of quinolones on crown ether CSP: Thermodynamics, chiral discrimination mechanism and application in biological samples. Journal of Chromatography B 1166, 122550

Clayden, Jonathan; Greeves, Nick; Warren, Stuart (2012). Organic Chemistry (2nd ed.). Oxford University Press. pp. 91. ISBN 978-0-19-927029-3.

Housecroft, C. E.; Sharpe, A. G. (2012). Inorganic Chemistry (4th ed.). Prentice Hall. pp. 35-37. ISBN 978-0-27374275-3.

IUPAC, 1997. Compendium of Chemical Terminology, $2^{\text {nd }}$ ed. (the "Gold Book"). Online corrected version: (2006) "Bond number". doi:10.1351/goldbook.B00705.

March, J., 1985., Advanced Organic Chemistry: Reactions, Mechanisms, and Structure (3rd ed.), New York: Wiley, ISBN 0-471-85472-7

Suhail, M., Ali I., 2020a. A magic trick for determination of ground state term of s, p \& d-orbital electrons. Chemistry International 6(4), 218-223.

Suhail, M., 2020b. A novel projection for anomers of glucose. Chemistry International 6(4), 301-304.
Suhail, M., Danish, S.M., Ali, I., Ansari, A., Arora, S., 2020c. Theoretical DFT study of Cannizzaro reaction mechanism: A mini perspective European Journal of Chemistry 11(2), 139-144.

Visit us at: http://bosaljournals.com/chemint/ Submissions are accepted at: editorci@bosaljournals.com 